Prepared for the Department of Homeland Security, Science and Technology Directorate under Contract AGRHSHQDC08X00571/B3

\title{
Solvent Extraction of Chemical Attribution Signature Compounds from Painted Wall Board: Final Report
}

JH Wahl

HA Colburn

October 2009

Pacific Northwest

NATIONAL LABORATORY

Proudly Operated by Battelle Since 1965 


\title{
DISCLAIMER
}

This report was prepared as an account of work sponsored by an agency of the United States Government. Neither the United States Government nor any agency thereof, nor Battelle Memorial Institute, nor any of their employees, makes any warranty, express or implied, or assumes any legal liability or responsibility for the accuracy, completeness, or usefulness of any information, apparatus, product, or process disclosed, or represents that its use would not infringe privately owned rights. Reference herein to any specific commercial product, process, or service by trade name, trademark, manufacturer, or otherwise does not necessarily constitute or imply its endorsement, recommendation, or favoring by the United States Government or any agency thereof, or Battelle Memorial Institute. The views and opinions of authors expressed herein do not necessarily state or reflect those of the United States Government or any agency thereof.

\author{
PACIFIC NORTHWEST NATIONAL LABORATORY \\ operated by \\ BATTELLE \\ for the \\ UNITED STATES DEPARTMENT OF ENERGY \\ under Contract DE-AC05-76RL01830 \\ Printed in the United States of America \\ Available to DOE and DOE contractors from the \\ Office of Scientific and Technical Information, \\ P.O. Box 62, Oak Ridge, TN 37831-0062; \\ ph: (865) 576-8401 \\ fax: (865) 576-5728 \\ email: reports@adonis.osti.gov

\footnotetext{
Available to the public from the National Technical Information Service, U.S. Department of Commerce, 5285 Port Royal Rd., Springfield, VA 22161 ph: (800) 553-6847 fax: (703) 605-6900

email: orders@ntis.fedworld.gov

online ordering: http://www.ntis.gov/ordering.htm
}

This document was printed on recycled paper. 


\section{Solvent Extraction of Chemical Attribution Signature Compounds from Painted Wall Board: Final Report}

JH Wahl

HA Colburn

October 2009

Prepared for the

Department of Homeland Security, Science and Technology Directorate under Contract AGRHSHQDC08X00571/B3

Pacific Northwest National Laboratory

Richland, Washington 99352 



\begin{abstract}
This report summarizes work that developed a robust solvent extraction procedure for recovery of chemical attribution signature (CAS) compound dimethyl methyl phosphonate (DMMP) (as well as diethyl methyl phosphonate (DEMP), diethyl methyl phosphonothioate (DEMPT), and diisopropyl methyl phosphonate (DIMP)) from painted wall board (PWB), which was selected previously as the exposed media by the chemical attribution scientific working group (CASWG). An accelerated solvent extraction approach was examined to determine the most effective method of extraction from PWB. Three different solvent systems were examined, which varied in solvent strength and polarity (i.e., 1:1 dichloromethane : acetone, $100 \%$ methanol, and $1 \%$ isopropanol in pentane) with a 1:1 methylene chloride : acetone mixture having the most robust and consistent extraction for four original target organophosphorus compounds. The optimum extraction solvent was determined based on the extraction efficiency of the target analytes from spiked painted wallboard as determined by gas chromatography $\mathrm{x}$ gas chromatography mass spectrometry (GCxGC-MS) analysis of the extract. An average extraction efficiency of approximately $60 \%$ was obtained for these four compounds. The extraction approach was further demonstrated by extracting and detecting the chemical impurities present in neat DMMP that was vapor-deposited onto painted wallboard tickets.
\end{abstract}





\section{Acronyms, Abbreviations, and Initialisms}

ASE

CAS

CASWG

CWA

DEMP

DEMPT

DIMP

DMMP

SNL

SOP

PNNL

PWB accelerated solvent extraction

chemical attribution signature

chemical attribution scientific working group

chemical warfare agent

diethyl methyl phosphonate

diisopropyl methyl phosphonate

diisopropyl methyl phosphonate

dimethyl methyl phosphonate

Sandia National Laboratory

standard operating procedure

Pacific Northwest National Laboratory

painted wall board 



\section{Contents}

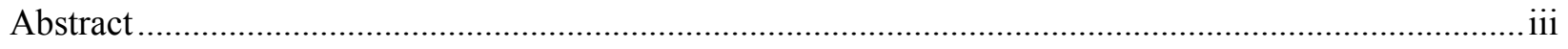

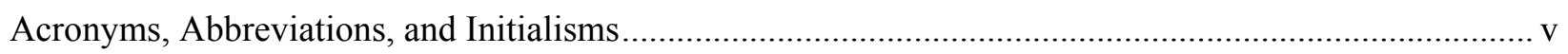

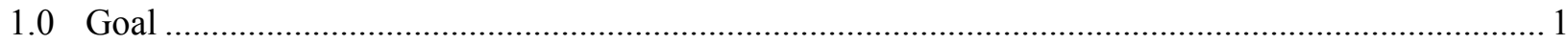

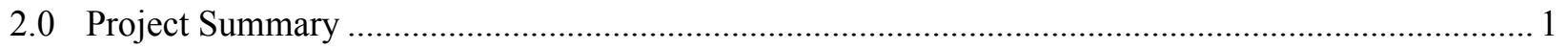

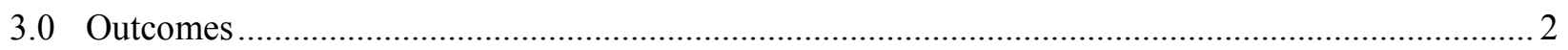

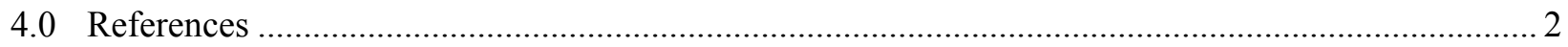

Appendix A - Pressurized Fluid Extraction Method for Painted Wall Board ........................................ A.1

Appendix B - Extraction of Chemical Impurities for Forensic Investigations: A Case Study for

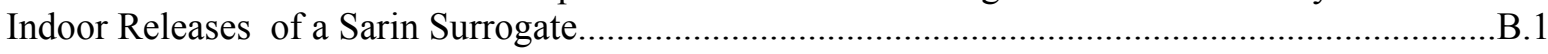





\subsection{Goal}

The goal of this project was to develop and characterize an analytical extraction procedure for recovery of chemical attribution signature (CAS) compound dimethyl methyl phosphonate (DMMP) from painted wall board (PWB), which was previously vetted by the chemical attribution scientific working group (CASWG).

\subsection{Project Summary}

This report summarizes the work performed involving the development and characterization of the analytical procedure for the recovery of CAS compounds. The persistence of a CAS in an indoor environment is influenced by the available chemical collection reservoirs and sinks that are present in an environment and that chemically partition with a CAS component. These reservoirs fall into two general categories - those that possess a large amount of absorbent, and those that possess a large adsorbent surface. A painted surface can have both of these properties. Paints are widely used in residential and commercial buildings, and the surface areas covered by this kind of coating are very large and thus very likely to be exposed to a building-related event. Moreover, the painted layer and the substrate supporting the paint can behave as an ab(d)sorbent material (Karlsson 1996, Munro 1999, Feng 2006). As a result, PWB provides an excellent reservoir for CAS collection post event. The challenge is to extract and detect the CAS components in an analytically robust and reproducible manner. This work is built upon the scenario of a sarin release in an office building, which was previously determined by the CASWG. This work developed and characterized a solvent extraction procedure for the recovery of CAS compounds from PWB.

Solvent extraction techniques coupled with separation and detection methods are widely used and proven techniques for effectively removing and capturing chemical warfare agent (CWA)-related compounds from materials. Because of the wide range in polarity and volatility of CAS compounds, solvent extraction is a logical starting point for extraction of materials to enable the detection and identification of CAS compounds and their degradation products. Substantial expertise exists at Pacific Northwest National Laboratory (PNNL) for using accelerated solvent extraction (ASE) for CWA and related compounds. ASE is a pressurized solvent extraction technique that utilizes solvents at elevated temperatures and pressures to accelerate the chemical extraction process from materials. The higher temperature increases the extraction kinetics, while the elevated pressure prevents the solvent from boiling. The hot liquid solvent has greatly improved solvating power and extraction efficiency. ASE is a common commercially available technique and is simple and fast to utilize.

This analytical extraction procedure was developed and characterized for the recovery of DMMP (in addition to diethyl methyl phosphonate (DEMP), diethyl methyl phosphonothioate (DEMPT), and diisopropyl methyl phosphonate (DIMP)) from PWB. An accelerated solvent extraction approach was examined where three different solvent systems were examined varying in solvent strength and polarity (i.e., 1:1 dichloromethane : acetone, $100 \%$ methanol, and 1\% isopropanol in pentane). The 1:1 methylene chloride : acetone mixture produced the most robust and consistent extraction for four original target organophosphorus compounds. The optimum extraction solvent was determined based on the extraction efficiency of the target analytes from spiked painted wallboard as determined by gas chromatography $\mathrm{x}$ 
gas chromatography mass spectrometry (GCxGC-MS) analysis of the extract. An average extraction efficiency of approximately $60 \%$ was obtained for these four compounds. The extraction approach was further demonstrated by extracting and detecting the chemical impurities present in neat DMMP that was vapor-deposited onto painted wallboard tickets supplied by Sandia National Laboratory (SNL).

\subsection{Outcomes}

The result of this project includes a standard operating procedure (SOP) Pressurized Fluid Extraction Method for Painted Wall Board that was drafted, sent to the client for review, and is attached to this document as Appendix A. In addition, a journal article, "Extraction of Chemical Impurities for Forensic Investigations: A Case Study for Indoor Releases of a Sarin Surrogate,” was drafted, sent to the client, submitted to Building and Environment, and is attached in this document as Appendix B.

\subsection{References}

Feng L, J Niu and L Zhang. 2006. "A physically-based model for prediction of VOCs emissions from paint applied to an absorptive substrate," Building and Environment, 41:1317-1325.

Karlsson E and U Huber. 1996. "Influence of desorption on the indoor concentration of toxic gases," J. Hazard. Mater., 49:15-27.

Munro NB, SS Talmage, GD Griffin, LC Waters, AP Watson, JF King and V Hauschild. 1999. "The sources, fate, and toxicity of chemical warfare agent degradation products [Review]," Environ. Health. Perspect., 107:933-974. 


\section{Appendix A}

\section{Pressurized Fluid Extraction Method for Painted Wall Board}





\section{Appendix A}

\section{Pressurized Fluid Extraction Method for Painted Wall Board}

Prepared by: Jon H. Wahl and Heather A. Colburn

Prepared at: Pacific Northwest National Laboratory

Introductory Notes/Comments:

This standard analytical method described herein relies heavily on the U.S. EPA SW-846, Test Methods for Evaluating Solid Waste, Physical/Chemical Methods. The SW-846 is an official compendium of analytical and sampling methods that have been evaluated and approved for use in complying with the Resource Conservation and Recovery Act (RCRA) regulations. SW-846 functions primarily as a guidance document setting forth acceptable, although not required, methods for the regulated and regulatory communities to use in responding to RCRA-related sampling and analysis requirements. The procedure discussed in this standard analytical method follows the method as described in U.S. EPA Method 3545A, Pressurized Fluid Extraction and Method.

\begin{tabular}{|l|l|l|}
\hline $\begin{array}{c}\text { Approval, } \\
\text { Concurrence or } \\
\text { Review: }\end{array}$ & \multicolumn{2}{c|}{ Dame \& Title/Position } \\
\hline$\square$ Approval & & \\
$\square$ Review & & \\
$\square$ Concurrence & & \\
\hline$\square$ Approval & & \\
$\square$ Review & & \\
$\square$ Concurrence & & \\
\hline$\square$ Approval & & \\
$\square$ Review & & \\
$\square$ Concurrence & & \\
\hline$\square$ Approval & & \\
$\square$ Review & & \\
$\square$ Concurrence & & \\
\hline$\square$ Approval & & \\
$\square$ Review & & \\
$\square$ Concurrence & & \\
\hline
\end{tabular}




\section{SCOPE AND APPLICATION:}

1.1 This method is a procedure for extracting a variety of organophosphorus compounds (specifically dimethyl methyl phosphonate, DMMP) from painted wall board sections and likely other dry solids. The procedure discussed in this standard analytical method follows the method as described in U.S. EPA Method 3545A, Pressurized Fluid Extraction and Method and 3500C, Organic Extraction and Sample Preparation. This method uses elevated temperature $\left(75^{\circ} \mathrm{C}\right)$ and pressure (1500-2000 psi) to achieve analyte recoveries. This procedure was developed on a commercially available, automated extraction system.

1.2 Use of this method is restricted to use by, or under the supervision of, appropriately experienced and trained personnel. Each analyst must demonstrate the ability to generate acceptable results with this method.

\section{SUMMARY OF METHOD:}

2.1 Samples are prepared for extraction by cutting a PWB section to $\sim 5 / 8 \times 3$ inch dimensions. The sample is then loaded into the extraction cell.

WARNING: Grinding is not recommended, due to safety concerns and it may also be a concern for other more volatile analytes.

2.2 The extraction cell containing the sample is heated to the extraction temperature, pressurized with the appropriate solvent system, and extracted for 5-10 minutes.

2.3 The solvent is collected away from the heated extraction vessel and allowed to cool. Since the extraction cells contain frits, no filtration of the extracts is needed.

2.4 The extract may be concentrated, if necessary for the determinative step being employed.

\section{INTERFERENCES:}

3.1 Solvents, reagents, glassware, and other sample processing hardware may yield artifacts and/or interferences to sample analysis. All of these materials must be demonstrated to be free from interferences under the conditions of the analysis by analyzing method blanks.

3.2 Interferences coextracted from the samples will vary considerably from source to source. If analysis of an extracted sample is prevented due to interferences, further cleanup of the sample extract may be necessary.

\section{SAFETY:}

4.1 This method does not address all safety issues associated with its use. The laboratory is responsible for maintaining a safe work environment and a current awareness file of OSHA regulations regarding the safe handling of the chemicals listed in this method. A reference file of material safety data sheets (MSDSs) should be available to all personnel involved in these analyses. 
4.2 The use of organic solvents, elevated temperatures, and high pressures in this method present potential safety concerns in the laboratory. Common sense laboratory practices can be employed to minimize these concerns.

4.3 Extraction cells in the oven are hot enough to burn unprotected skin. Allow the cells to cool for 10-15 min before removing them from the oven or use appropriate protective equipment (e.g., insulated gloves or tongs), as recommended by the manufacturer.

4.4 During the gas purge step, some solvent vapors may exit through a vent port in the instrument. Follow the manufacturer's directions regarding connecting this port to a fume hood or other means to prevent release of solvent vapors to the laboratory atmosphere.

4.5 The instrument may contain flammable vapor sensors and should be operated with all covers in place and doors closed to ensure proper operation of the sensors. Follow the manufacturer's directions regarding replacement of extraction cell seals to ensure against vapor leaks.

\section{APPARATUS:}

The mention of trade names or commercial products in this SOP does not constitute an endorsement or exclusive recommendation for use. Glassware, reagents, supplies, equipment, and settings other than those listed in this method may be employed provided that method performance appropriate for the intended application has been demonstrated and documented.

This section does not list common laboratory glassware (e.g., beakers and flasks).

\subsection{Pressurized fluid extraction device}

5.1.1 Dionex Accelerated Solvent Extractor ${ }^{\circledR}$ with appropriately-sized extraction cells. Currently, cells are available that will accommodate 1-g, 5-g, 10-g, 20-g and 30-g samples. Cells should be made of stainless steel or other material capable of withstanding the pressure levels $(2000+$ psi) necessary for this procedure.

5.1.2 Other system designs may be employed, provided that adequate performance can be demonstrated for the analytes and matrices of interest.

5.2 Device for cutting painted wall board to desired dimensions such that the pieces fit into the extraction cell.

5.3 Analytical balance, capable of weighing to $0.01 \mathrm{~g}$.

5.4 Vials for collection of extracts -- 40-mL or 60-mL, pre-cleaned, open-top screw-cap with polytetrafluoroethylene (PTFE)-lined silicone septum (e.g., Dionex 049459, 049460, 049461, 049462 or equivalent).

5.5 Filter disk -- appropriate cellulose or glass fiber (e.g., Dionex 049458 or 047017, or equivalent).

5.6 Appropriate extraction cell and end caps (depend upon available Dionex system). 
6. REAGENTS AND MATERIALS:

6.1 Reagent-grade chemicals must be used in all tests. Unless otherwise indicated, it is intended that all reagents conform to the specifications of the Committee on Analytical Reagents of the American Chemical Society, where such specifications are available. Other grades may be used, provided it is first ascertained that the reagent is of sufficiently high purity to permit its use without lessening the accuracy of the determination. Reagents should be stored in glass to prevent the leaching of contaminants from plastic containers.

6.2 Quartz sand -- Clean sand may be used to fill void volumes in the extraction cell. Sand with a small particle size should not be used, as the fine particles can stick to the threads of the cell, resulting in leaks and damage to the threads. Ottawa sand, with a particle size of 20-30 mesh, has been found to work well and it is readily available from suppliers (e.g., Fisher Scientific, S23-3, or equivalent). If sand is utilized as a filler, it should be cleaned by heating at $400^{\circ} \mathrm{C}$ for $4 \mathrm{hrs}$ in a shallow tray.

\subsection{Extraction solvents}

All solvents should be pesticide quality or equivalent. Solvents may be degassed prior to use.

6.4 Organophosphorus compounds are extracted with an acetone/methylene chloride $(1: 1, \mathrm{v} / \mathrm{v})$, $\mathrm{CH}_{3} \mathrm{COCH}_{3} / \mathrm{CH}_{2} \mathrm{Cl}_{2}$.

6.5 High-purity gases such as nitrogen, carbon dioxide, or helium are used to purge and/or pressurize the extraction cell. Follow the instrument manufacturer's recommendation for the choice of gases.

\section{SAMPLE COLLECTION, PRESERVATION AND HANDLING:}

7.1 Samples to be extracted by this procedure should be collected and stored like any other solid samples containing semivolatile organics.

\section{STANDARDS AND CONTROLS:}

8.1 Any effort involving the collection of analytical data should include development of a systematic planning document, such as a Quality Assurance Project Plan (QAPP) or a Sampling and Analysis Plan (SAP). The laboratory should maintain a formal quality assurance program. The laboratory should also maintain records to document the quality of the data generated. All data sheets and quality control data should be maintained for reference or inspection.

8.2 Initial demonstration of proficiency

The laboratory must demonstrate initial proficiency with sample preparation and determinative method combination it utilizes by generating data of acceptable accuracy and precision for target analytes in a clean matrix. The laboratory must also repeat the demonstration of proficiency whenever new staff are trained or significant changes in instrumentation are made.

8.3 Initially, before processing any samples, the analyst should demonstrate that all parts of the equipment in contact with the sample and reagents are interference-free. For this method, this can be accomplished through the analysis of a solid matrix method blank (e.g., clean sand). As a continuing check, each time samples are extracted, cleaned up, and analyzed, and when there is a 
change in reagents, a method blank should be prepared and analyzed for the compounds of interest as a safeguard against chronic laboratory contamination.

8.4 Any method blanks, matrix spike samples, or replicate samples should be subjected to the same analytical procedures as those used on actual samples.

8.5 Standard quality assurance practices should be used with this method as included in appropriate systematic planning documents and laboratory SOPs. All instrument operating conditions should be recorded.

\section{CALIBRATION:}

9.1 There are no calibration or standardization steps directly associated with this sample extraction procedure, other than establishing the extraction conditions.

\section{SAMPLING:}

10.1 There are no specific sampling steps directly associated with this sample extraction procedure, other than obtaining a section of wall board for subsequent subsampling for extraction.

\section{OTHER QUALITY ASSURANCE CONSIDERATIONS:}

11.1 There are no calculations explicitly associated with this extraction procedure. See the appropriate determinative method for the calculation of final sample results.

\section{PROCEDURE (Step-by-Step Directions)}

\subsection{Sample preparation}

Pressurized fluid extraction performs best on dry, finely-ground solids; however, the processes of sample drying and grinding involve the potential for a loss of analytes, the introduction of other contaminants into the sample, the contamination of the laboratory environment, and exposure of the analyst to environmental contaminants.

12.1.1 Cut a portion of the wall board to fit into the extraction cell (e.g., $\sim 5 / 8 \times 3$ inches for a $22 \mathrm{~mL}$ extraction cell)

\subsection{Weighing the sample}

Weigh the appropriate aliquot of the sample needed for the determinative method and the sensitivity needed for the project into the extraction cell of the appropriate size.

NOTE: The weight of a specific sample that a cell will contain depends on the bulk density of the sample. Generally, an 11-mL cell will hold about $10 \mathrm{~g}$ of material, a 22-mL cell will hold about $20 \mathrm{~g}$ of material, and a $33-\mathrm{mL}$ cell will hold about $30 \mathrm{~g}$ of material. Analysts should ensure that the sample aliquot extracted is large enough to provide the necessary sensitivity and choose the extraction cell size accordingly.

If the sample is weighed in the extraction cell, then prepare the cell by placing a disposable cellulose or glass fiber filter in the cell outlet before adding the sample. Record the weight of the 
empty cell and the weight of the sample and the cell. Determine the weight of the sample by difference.

\subsection{Adding surrogates and other spiked compounds}

Add the surrogates (or labeled internal standards) listed in the determinative method to each sample in its extraction cell or in another clean container.

CAUTION: It is critical that any compounds added to a sample, including the surrogates, are added to the sample aliquot prior to any additional processing steps. It is also critical that the spiked compounds be in the same chemical form as the target compounds.

12.4 Clean sand may be used to fill any void volume in the extraction cells. Follow the manufacturer's recommendations regarding the need to fill void volumes.

NOTE: Since the sand is not intended to dry the sample, it need not be mixed with the sample, but can be placed into the cell.

12.5 Seal the extraction cell according to the manufacturer's instructions. Use disposable cellulose or glass fiber filters in the cell outlets. Place the extraction cell into the instrument or autosampler tray, as described by the instrument manufacturer.

12.6 Place a precleaned collection vessel in the instrument for each sample, as described by the instrument manufacturer. The total volume of the collected extract will depend on the specific instrumentation and the extraction procedure recommended by the manufacturer and may range from 0.5 to 1.4 times the volume of the extraction cell. Ensure that the collection vessel is sufficiently large to hold the extract.

NOTE: The volume of solvent used for each extraction is a function of the size of the extraction cell, not the weight of the sample. Consult the manufacturer's instructions for the appropriate volume of solvent to employ for a given cell size and the necessary collection vessel volume.

\subsection{Recommended extraction conditions}

Oven temperature: $75^{\circ} \mathrm{C}$

Pressure: $1500-2000$ psi

Static time: 5 min (after 5-min pre-heat equilibration)

Flush volume: $60 \%$ of the cell volume

Nitrogen purge: $60 \mathrm{sec}$ at $150 \mathrm{psi}$ (purge time may be extended for larger cells)

Static cycles: 2

NOTE: In general, the pressure is not a critical parameter, as the purpose of pressurizing the extraction cell is to prevent the solvent from boiling at the extraction temperature and to ensure that the solvent remains in intimate contact with the sample. Any pressure in the range of 1500-2000 psi should suffice.

12.8 Begin the extraction according to the manufacturer's instructions.

12.9 Collect each extract in a clean vial. Generally, the extracts are near room temperature upon collection. 
12.10 The extract is now ready for concentration and analysis, depending on the extent of interferants and the determinative method to be employed.

\section{METHOD PERFORMANCE:}

Performance criteria should be developed on a project-specific basis, and the laboratory should establish in-house QC performance criteria for the application of this method. These performance data are not intended to be and must not be used as absolute QC acceptance criteria for purposes of laboratory accreditation.

\subsection{Organophosphorus compounds}

The extraction procedure discussed in this standard analytical method follows the method as described in U.S. EPA Method 3545A, Pressurized Fluid Extraction and Method. U.S EPA Method 8141B, Organophosphorus Compounds by Gas Chromatography, provides the gas chromatographic procedure for determination of organophosphorus compounds. General method performance is summarized in U.S. EPA Method 8270D (Semivolatile Organic Compounds by Gas Chromatography/Mass Spectrometry (GC/MS)) and 8081B (Organochlorine Pesticides by Gas Chromatography). Specifically the recovery for DMMP utilizing this standard analytical method against painted wall board was found to $\sim 60 \%$, which is comparable to the organophosphorous compounds listed in Reference 3.

\section{REFERENCES}

1. U.S. Environmental Protection Agency. U.S. EPA Method 3545A, Pressurized Fluid Extraction, contained in "Test Methods for Evaluating Solid Waste, Physical/Chemical Methods," SW-846, http://www.epa.gov/osw/hazard/testmethods/sw846/online/index.htm

2. U.S. Environmental Protection Agency. U.S. EPA Method 3500C, Organic Extraction and Sample Preparation, contained in "Test Methods for Evaluating Solid Waste, Physical/Chemical Methods," SW-846, http://www.epa.gov/osw/hazard/testmethods/sw846/online/index.htm

3. "Extraction of Organophosphorus Pesticides Using Accelerated Solvent Extraction (ASE)," Application Note 319, Dionex Corporation, 2004.

4. Julia Popa, Fariborz Haghighat, "The impact of VOC mixture, film thickness and substrate on adsorption/desorption characteristics of some building materials," Building and Environment, 38 (2003) 959-964.

5. Feng Li, Jianlei Niu, Lizhi Zhang, "A physically-based model for prediction of VOCs emissions from paint applied to an absorptive substrate," Building and Environment, 41 (2006) 1317-1325. 



\section{Appendix B}

Extraction of Chemical Impurities for Forensic Investigations:

A Case Study for Indoor Releases

of a Sarin Surrogate 



\section{Appendix B}

\section{Extraction of Chemical Impurities for Forensic Investigations: A Case Study for Indoor Releases of a Sarin Surrogate}

Heather A. Colburn and Jon H. Wahl*

Chemical and Biological Sciences Group, Pacific Northwest National Laboratory, Richland, WA 99352, USA

*Pacific Northwest National Laboratory

902 Battelle Boulevard

P.O. Box 999, MSIN P8-50

Richland, WA 99352 USA

Tel: 1-509-371-6677

Fax: 1-509-372-4583

jon.wahl@pnl.gov

Submitted as a technical note to Building and Environment. 


\begin{abstract}
A solvent extraction approach was developed and examined for extraction of targeted organophosphorus compounds as well as nerve agent simulants from painted wallboard (PWB). Painted wallboard was chosen as a substrate due to its presence as large surface area media in an indoor environment that is applicable to a chemical agent release scenario. Three different solvent systems were examined with a 1:1 methylene chloride : acetone mixture having the most robust and consistent extraction for four target organophosphorus compounds (dimethyl methyl phosphonate (DMMP), diethyl methyl phosphonate (DEMP), diethyl methyl phosphonothioate (DEMPT), and diisopropyl methyl phosphonate (DIMP)). An average extraction efficiency of approximately $60 \%$ was obtained for these four compounds. The extraction approach was further demonstrated by extracting and detecting the chemical impurities present in neat DMMP that was vapor deposited onto painted wallboard tickets as a simulant to an agent release. Five chemical impurities that were present in DMMP - dimethyl phosphate, trimethyl ester phosphoric acid, ethyl methyl methylphosphonate, O,O,S-trimethyl ester phosphorothioic acid, and biphenyl- were detected on the PWB and were utilized to determine the source/supplier of the DMMP.
\end{abstract}

Keywords: volatile organic compounds, chemical extraction, surrogate, wallboard, building contamination 


\section{Introduction}

After an intentional chemical release (e.g., nerve agent) in a building many residual components remaining in a chemical formulation (i.e., impurities) may provide valuable criminal investigative clues. These key components may be due to unreacted reagents, degradation products, side-reaction products, or impurities in the original chemical and may help with discovering various manufactures or processes utilized for production or may help with matching various samples to each other. All indoor materials interact with the airborne chemicals. These materials or surfaces can act as sorbents, which can behave as a chemical sink/sponge as well as a source of volatile organic compound (VOC) emission. Absorption of airborne compounds may allow the chemicals to persist for subsequent chemical analysis, which could be vital to criminal investigation. The sorption properties of various VOCs on common indoor materials have been investigated by various approaches and under various conditions [e.g., refs 1-20]. The sorption properties of the chemical nerve agent sarin has been studied by Karlsson and Huber [21] utilizing a single sink model to estimate sorption and desorption rates of sarin on surfaces. Blewett and Arca [22] reported experiments in which a small cottage was exposed to exterior concentrations of 5-7 $\mathrm{g} \mathrm{m}^{-3}$ sarin over a 1 hour period in a larger test chamber. When developing methods that will ultimately be used with restricted or highly toxic chemicals, such as chemical nerve agents, a surrogate compound is often utilized. For example, G-type nerve agents have been studied with surrogates having similar reactivity or functional groups $[23,24]$. A commonly used surrogate for sarin is dimethyl methyl phosphonate (DMMP), where the fluorine atom and one isopropyl group present in sarin are substituted with two methoxy groups. Alternately, diisopropyl methyl phosphonate (DIMP) can also be utilized, where this compound retains the isopropoxy group, but a second isopropyl group is substituted for the fluorine atom. Singer et.al [25] determined the sorption rate parameters for three organophosphorus (OP) compounds [dimethyl methylphosphonate (DMMP), diethyl ethylphosphonate (DEEP), and triethyl phosphate (TEP)] as surrogates for the G-type nerve agents sarin (GB), soman (GD), and tabun (GA).

This paper describes a method to extract chemicals from painted wallboard and examination of the extraction efficiency of that method for a variety of organophosphate compounds and possible 
component impurities. Painted wallboard was chosen as a substrate due to its large surface area present in a typical office indoor environment and due to its potential sorbent capability (i.e., chemical sink) for chemical compounds. Popa and Haghighat [26] demonstrated that painted gypsum board adsorbs both toluene and mixtures of VOCs at higher rates than when paint was applied on aluminum and that as the thickness of paint increases the sorption strength increases. Li and coworkers developed a model that indicates that substrates like particle board can act as "chemical sponge", which decreases peak component concentrations and extends the presence and emission of VOCs [27]. The extraction approach of the target compounds in this work is based upon pressurized fluid extraction utilizing a Dionex accelerated solvent extraction (ASE) system. In this approach the sample (i.e., painted wallboard) is placed within an extraction cell and solvent is pumped into the cell. The cell is then isolated and the system is brought to an elevated temperature and pressure for extraction. Subsequently the extract is removed and transferred for analysis or further cleanup. Both DMMP and DIMP were contained within the target compound set used in this study. In addition residual trace impurities present from the chemical production process were also found.

\section{Experimental Section}

\section{Chemicals}

The extraction solvents pentane, methanol, methylene chloride, and acetone, were all pesticide residue grade or better and were purchased from Sigma-Aldrich (St. Louis, MO, USA). Toluene and isopropanol were purchased from Burdick and Jackson (Morristown, NJ, USA) and JT Baker (Phillipsburg, NJ, USA) respectively and were HPLC grade or better. Dimethyl methylphosphonate (DMMP), diethyl methylphosphonate (DEMP), diisopropyl methylphosphonate (DIMP), diethyl, methylphosphonothioate (DEMPT) were all purchased from Sigma-Aldrich (St. Louis, MO, USA). Standard stock solutions of $10 \mathrm{mg} / \mathrm{mL}$ were prepared in pentane or toluene. From the stock solutions, the four compounds were combined and diluted in toluene for calibration standards as well as spiking onto the painted wallboard substrate for extraction method development. 


\section{Painted Wallboard Samples}

PNNL PWB

Excess wallboard (i.e., gypsum drywall) was obtained from a new house of one of the authors and was painted utilizing a 3inch bristle paint brush. One coat of Kilz primer (Masterchem Industries, Imperial, MO, USA) was applied by brush followed by one coat of white ceiling paint (Parker Paint, Tacoma, WA, USA). This afforded a large supply of PWB to develop and examine the extraction process. Although no construction and painting specification or standards were followed for this PWB supply the procedure was considered adequate as a typical painting approach was utilized.

\section{Sandia National Laboratory PWB}

The PWB obtained from Sandia National Laboratory (SNL) was prepared in accordance with Sandia Construction Standard Specifications Section 09250, Gypsum Drywall, and Section 09900, Painting. 6x6 inch PWB tickets were prepared by aerosol application of a $0.7 \mathrm{wt} \%$ dimethyl methyl phosphonate (DMMP) solution in HPLC grade hexanes (Sigma-Aldrich, St. Louis, MO, USA). These tickets were stored at PNNL for approximately one year at $-80^{\circ} \mathrm{C}$ until they were examined.

\section{Pressurized Fluid Extraction (PFE)}

A Dionex (Dionex Corporation, Sunnyvale, CA, USA) accelerated solvent extraction (ASE) system 200 was utilized for compound extraction from the painted wallboard. The system utilizes solvents at high temperatures and pressures to accelerate the chemical extraction process. Pieces of PWB were coarsely ground in a mortar and pestle or extracted whole by cutting the wallboard into 5/8" x 3 " pieces with a small hacksaw. The ASE extraction cells were $22 \mathrm{~mL}$ in volume, glass fiber extraction cell filters were used. The wall board was extracted at $75^{\circ} \mathrm{C}, 2000 \mathrm{psi}$, static time of $5 \mathrm{~min}$, flush volume $60 \%, 2$ cycles. The resulting extraction volume, $\sim 30 \mathrm{~mL}$, was then reduced to $\sim 1 \mathrm{~mL}$ utilizing a Caliper TurboVap II (Caliper Life Sciences, Hopkinton, MA, USA). The final sample was stored at $-20^{\circ} \mathrm{C}$ until 
analyzed. If solid pieces were extracted, 20-30 mesh size Ottawa sand purchased from Thermo Fisher Scientific (Pittsburgh, PA, USA) was used to fill the void spaces in the extraction cells.

\section{Chemical Analysis}

Chemical analysis of the PWB extracts were performed on a Leco Pegasus 4D GCxGC-MS system (LECO Corp., St. Joseph, MI, USA) equipped with a Gerstel multipurpose sampler (MPS2) (Gerstel Inc., Baltimore, MD, USA). Two-dimensional gas chromatography (GCxGC) is a multidimensional separation technique that separates a sample sequentially on two different columns. Two columns with different specificities are chosen to separate mixtures on distinct chemical properties. In general GCxGC-MS provides greatly enhanced separations and improved sensitivity, which is comparable to typical single ion monitoring approaches, but collects the full mass spectral acquisition range. The separation utilized ultra-high purity helium as the carrier gas, set at $1.5 \mathrm{~mL} / \mathrm{min}$, constant flow. Unless stated otherwise the experimental conditions are listed in Table 1.

\section{Results and Discussion}

An approach to extract chemicals from painted wallboard was developed for application to a homeland threat scenario of a nerve agent release in a high-rise office building. The optimum extraction solvent was determined based on the extraction efficiency of the target analytes from spiked ground painted wallboard as determined by gas chromatography $\mathrm{x}$ gas chromatography mass spectrometry (GCxGC-MS) analysis of the extract. The extraction efficiency for three different solvents was examined for the four target compounds DMMP, DEMP, DEMPT, and DIMP. Painted wallboard pieces were coarsely ground to maximize the possible surface area accessible for extraction and to more optimally fill the cylindrical ASE extraction cell. Six replicate ASE extractions were performed by liquid spiking $20 \mathrm{uL}$ of a $100 \mathrm{ng}$ / component / $\mathrm{uL}$ in toluene solution into the ground PWB contained in the extraction cell. Three different solvent systems were examined, which varied in solvent strength and polarity (i.e., 1:1 dichloromethane : acetone, $100 \%$ methanol, and 1\% isopropanol in pentane). After extraction the 
recovered extract was concentrated down to $1 \mathrm{~mL}$ utilizing a Caliper TurboVap II. One microliter was injected splitless and analyzed utilizing the GCxGC-MS system. Consequently, approximately 2ng/component was injected on-column assuming $100 \%$ extraction efficiency. Typical 2D total ion chromatograms (TICs) obtained from extracted PWB are illustrated in Figure 1 for the three solvent extraction systems. Within the chromatograms depicted in Figure 1 hundreds of components can be found, where the large solvent front from the splitless injection is shown early in the separations while in the later portion of the separation ( $>2500 \mathrm{sec}$ 1D) are large alkyl components that are typically found within paint products (e.g., >C20 type alkyl compounds). The circled region in Figure 1 within the 1:1 methylene chloride : acetone extract highlights the region where the four target compounds elute within the separation. This target region is examined in greater detail in Figure 2 where the three chromatograms shown are reconstructed single ion chromatograms (SICs) utilizing $\mathrm{m} / \mathrm{z}=79$ for clarity. In general all three ASE solvent systems extract the four target compounds as they can easily be found and identified within the separation. However, when $100 \%$ methanol is utilized many solute zones appear to have zone splitting. For example as shown in 100\% methanol chromatogram in Figure 2, DMMP and DEMP show multiple chromatographic zones and have identical mass spectra within their individual characteristic chromatographic regions. However, interestingly DEMPT with the addition of the sulfur atom to the compound shows a single chromatographic zone compared to the other target compounds. This solute zone splitting is likely due to a solvent-solute-stationary phase incompatibility. Methanol, being a very polar solvent, may be producing droplets along the stationary phase surface during the splitless injection process rather than a smooth flooded zone region required for correct solvent evaporation and solute focusing. Thus $100 \%$ methanol would likely not be an optimum extraction solvent for this reason alone unless a different, perhaps more polar, stationary phase was utilized.

The results for extraction efficiency for the four compounds are summarized in Figure 3, where the extracted amount of a component is illustrated as a function of the extraction solvent. The results determined for Figure 3 are based on the summed mass spectral ion signal intensities utilizing $\mathrm{m} / \mathrm{z}$ values $=79+94: 97+124+125+168$, which cover the mass spectral ions of the target compounds. Simple four 
point calibration curves, which encompassed the target extraction concentration exhibited $r^{2}=0.999$ for each component and were utilized to determine the extracted amount. In general the 1:1 methylene chloride : acetone solvent system offers the most consistent efficiency among the four compounds, with an average extraction efficiency of greater than $60 \%$. This extraction efficiency is comparable to that quoted for extraction of organophosphorus pesticides in soil matrices [28]. The large variability observed in Figure 3 is likely due to the heterogeneity of the ground PWB per extraction cell and the actual placement of the liquid spike within the ground PWB. Whereas the $100 \%$ methanol solvent system depicts the greater recovery for DMMP (via summing split solute zones per component), the poor recovery for DEMPT and the problematic chromatographic solute zone splitting would make this solvent a poor choice. Conversely DMMP has poor recovery when $1 \%$ IPA : pentane is utilized, which is due to the poor solubility of DMMP in this relatively non-polar solvent system. As the number of alkyl groups within the compound increases for DEMP and DIMP extraction efficiency increases as compound solubility improves. These results illustrate that the 1:1 methlyene chloride : acetone is the best all around choice for extraction from PWB and was utilized in a further vapor exposure study.

\section{Examination of the Extraction Efficiency of Ground PWB vs. Whole pieces}

The extraction efficiency of whole pieces of PWB was compared to the extraction efficiency of ground material. Ground wall board was spiked as described above while the whole pieces of wall board were spiked on their painted surfaces, then inserted into extraction cells with Ottawa sand as an inert material to fill the open void spaces within the extraction cell. In both cases, approximately the same mass of PWB was extracted. Six replicates were prepared in each case. While the ground material had a greater extraction efficiency, the extraction efficiency from the solid pieces of material were approximately $50 \%$ in the 1:1 methylene chloride : acetone solvent. 


\section{Extraction of Impurities from Simulant-exposed PWB}

To further highlight this extraction approach, exposed PWB samples were prepared with vapor deposition of DMMP. The treated PWB tickets used for this study were obtained from SNL and were determined to have approximately $1 \mu \mathrm{g}$ DMMP $/ \mathrm{mm}^{2}$.vapor deposited onto $6 \mathrm{x} 6$ inch PWB tickets. Whereas the amount of DMMP per area is large, the goal was to offer and produce a high dose exposure of a simulant warfare agent in an office setting. A typical chromatogram obtained from the 1:1 methylene chloride : acetone extraction of the DMMP vapor exposed PWB is illustrated in Figure 4. Again hundreds of compounds can be found from this extraction and chemical analysis, most of which are related to the painted wallboard. DMMP is seen as a major extracted component in this case. However, a few components can be associated to impurities present in the neat DMMP; the region is highlighted in the rectangular area around DMMP in Figure 4. In related work at our laboratory, Hoggard and co-workers demonstrated that distinctive impurities present within DMMP can differentiate the six different manufactures or suppliers of DMMP [29]. In their work Hoggard and co-workers examined replicate analyses of the six DMMP samples utilizing GCxGC-MS and applied chemometric algorithms to differentiate samples. A portion of the chromatograms that distinguished the six neat DMMP sources utilized in Hoggards' study is illustrated in Figure 5. As can be seen in Figure 5, utilizing the selected $\mathrm{m} / \mathrm{z}$ values $(\mathrm{m} / \mathrm{z}=80 \mathrm{x} 0.05+111+154+156)$ to highlight the differences, the DMMP sources can be differenated from each other with the exception for DMMP 2 and 3. Utilizing this neat DMMP chromatographic data, five impurities (in addition to the absence of other impurities) were found in the extracted DMMP SNL PWB and appear to correlate to DMMP 1 referenced in Figure 5 and the work of Hoggard et.al. [29]. The comparison between DMMP 1 and the extracted SNL PWB is shown in Figure 6. These impurity fingerprints: dimethyl phosphonate, trimethyl ester phosphoric acid, ethyl methyl methylphosphonate, O,O,S-trimethyl ester phosphorothioic acid, and biphenyl were compared among their $2 \mathrm{D}$ retention pattern and their subsequent mass spectral information. Compound assignments are made with mass spectral similarity matches greater than 900 for the five compounds and are based upon NIST05 mass spectral database searches [30]. An advantage of the comprehensive gas chromatography - 
time-of-flight mass spectrometer is that full mass spectral range is obtained with sensitivity equal to or better than common single ion monitoring approaches.

\section{Conclusions}

An accelerated solvent extraction approach has been examined to determine the most effective method of extraction of chemical agent stimulants from PWB. Three different solvent systems were examined with a 1:1 methylene chloride : acetone mixture having the most robust and consistent extraction for four original target organophosphorus compounds. While ground material demonstrated superior extraction efficiency, extraction of the solid pieces of material was sufficient for extraction of all four of the test compounds. The solvent of choice also extracted known DMMP impurities from DMMP vapor-exposed painted wallboard. As demonstrated, this method is a valuable forensic investigative tool to aid in sourcing the material used in a chemical release.

\section{Acknowledgements}

Funding for this work was provided through contract AGRHSHQDC08X00571/B3to Pacific Northwest National Laboratory by the Department of Homeland Security, Science and Technology Directorate. The authors wish to thank Carlos Fraga at Pacific Northwest National Laboratory for the use of the DMMP samples and Dora Derzon and Theodore Borek at Sandia National Laboratory for the preparation of the vapor deposited wallboard tickets.

\section{References}

[1] Tichenor, B. A.; Guo, Z.; Dunn, J. E.; Sparks, L. E.; Mason, M. A. The interaction of vapour phase organic compounds with indoor sinks. Indoor Air 1991, 1, 23-35.

[2] Jorgensen, R. B.; Bjorseth, O. Sorption behaviour of volatile organic compounds on material surfaces - The influence of combinations of compounds and materials compared to sorption of single compounds on single materials. Environ. Int. 1999, 25, 17-27.

[3] Jorgensen, R. B.; Bjorseth, O.; Malvik, B. Chamber testing of adsorption of volatile organic compounds (VOCs) on material surfaces. Indoor Air 1999, 9, 2-9. 
[4] Jorgensen, R. B.; Dokka, T. H.; Bjorseth, O. Introduction of a sink-diffusion model to describe the interaction between volatile organic compounds (VOCs) and material surfaces. Indoor Air 2000, $10,27-38$.

[5] Colombo, A.; DeBortoli, M.; Knoppel, H.; Pecchio, E.; Vissers, H. Adsorption of selected volatile organic compounds on a carpet, a wall coating, and a gypsum board in a test chamber. Indoor Air 1993, 3, 276-282.

[6] Won, D.; Corsi, R. L.; Rynes, M. New indoor carpet as an adsorptive reservoir for volatile organic compounds. Environ. Sci. Technol. 2000, 34, 4193-4198.

[7] Won, D.; Corsi, R. L.; Rynes, M. Sorptive interactions between VOCs and indoor materials. Indoor Air 2001, 11, 246-256.

[8] Van Loy, M. D.; Lee, V. C.; Gundel, L. A.; Daisey, J. M.; Sextro, R. G.; Nazaroff, W. W. Dynamic behavior of semivolatile organic compounds in indoor air. 1. Nicotine in a stainless steel chamber. Environ. Sci. Technol. 1997, 31, 2554-2561.

[9] Van Loy, M. D.; Riley, W. J.; Daisey, J. M.; Nazaroff, W. W. Dynamic behavior of semivolatile organic compounds in indoor air. 2. Nicotine and phenanthrene with carpet and wallboard. Environ. Sci. Technol. 2001, 35, 560-567.

[10] an Der Wal, J. F.; Hoogeveen, A. W.; van Leeuwen, L. A quick screening method for sorption effects of volatile organic compounds on indoor materials. Indoor Air 1998, 8, 103-112.

[11] Tiffonnet, A. L.; Blondeau, P.; Allard, F.; Haghighat, F. Sorption isotherms of acetone on various building materials. Indoor Built Environ. 2002, 11, 95-104.

[12] Bouhamra, W.; Elkilani, A. Development of a model for the estimation of indoor volatile organic compounds concentration based on experimental sorption parameters. Environ. Sci. Technol. 1999, 33, 2100-2105.

[13] Bouhamra, W. S.; Elkilani, A. S. Investigation and modeling of surface sorption/desorption behavior of volatile organic compounds for indoor air quality analysis. Environ. Technol. 1999, 20, 531-545.

[14] Cox, S. S.; Zhao, D. Y.; Little, J. C. Measuring partition and diffusion coefficients for volatile organic compounds in vinyl flooring. Atmos. Environ. 2001, 35, 3823-3830.

[15] Meininghaus, R.; Kirchner, S.; Maupetit, F.; Sallee, H.; Quenard, D. Gravimetric studies on VOC adsorption by indoor materials under near-ambient conditions. Indoor Built Environ. 2000, 9, 277-283.

[16] Singer, B. C.; Revzan, K. L.; Hotchi, T.; Hodgson, A. T.; Brown, N. J. Sorption of organic gases in a furnished room. Atmos. Environ. 2004, 38, 2483-2494.

[17] Piade, J. J.; D'Andres, S.; Sanders, E. B. Sorption phenomena of nicotine and ethenylpyridine vapors on different materials in a test chamber. Environ. Sci. Technol. 1999, 33, 2046-2052. 
[18] Yang, X.; Chen, Q.; Zhang, J. S.; An, Y.; Zeng, J.; Shaw, C. Y. A mass transfer model for simulating VOC sorption on building materials. Atmos. Environ. 2001, 35, 1291-1299.

[19] Zhang, J.; Zhang, J. S.; Chen, Q.; Yang, X. A critical review on VOC sorption models. ASHRAE Trans. 2002, 108, 162-174.

[20] Zhang, J.; Zhang, J. S.; Chen, Q. Effects of environmental conditions on the VOC sorption by building materials Part II: Model evaluations. ASHRAE Trans. 2003, 109, 167-178.

[21] Karlsson, E.; Huber, U. Influence of desorption on the indoor concentration of toxic gases. $J$. Hazard. Mater. 1996, 49, 15-27.

[22] Blewett, W. K.; Arca, V. J. Experiments in sheltering in place: How filtering affects protection against sarin and mustard. Edgewood Chemical and Biological Directorate, Research and Technology Directorate, ECBC-TR-034; available via National Technical Information Service (www.ntis.gov), 1999.

[23] Munro, N. B.; Talmage, S. S.; Griffin, G. D.; Waters, L. C.; Watson, A. P.; King, J. F.; Hauschild, V. The sources, fate, and toxicity of chemical warfare agent degradation products [Review]. Environ. Health. Perspect. 1999, 107, 933-974.

[24] Zheng, F.; Zhan, C. G.; Ornstein, R. L. Theoretical studies of reaction pathways and energy barriers for alkaline hydrolysis of phosphotriesterase substrates paraoxon and related toxic phosphofluoridate nerve agents. J. Chem. Soc. Perkins Trans. 2 2001, 2355-2363.

[25] Singer, B.C.; Hodgson, A.R.; Destaillats, F.; Hotchi, R.; Revzan, K.L.; Sextro, R.G. Indoor Sorption of Surrogates for Sarin and Related Nerve Agents. Environ. Sci. Technol. 2005, 39, 3203-3214.

[26] Popa, J. and Haghighat, F., The impact of VOC mixture, film thickness and substrate on adsorption/desorption characteristics of some building materials. Building and Environment 2003, 38, $959-964$.

[27] Feng,L. Niu, J. Zhang, L., A physically-based model for prediction of VOCs emissions from paint applied to an absorptive substrate. Building and Environment, 2006, 41, 1317-1325.

[28] Extraction of Organophosphorus Pesticides Using Accelerated Solvent Extraction (ASE); Application Note 319, Dionex Corporation, 2004.

[29] Hoggard, J.C., Wahl, J.H., Synovec, R.E., Mong, G.M., Fraga, C.G., Impurity Profiling of a Chemical Weapon Precursor for Possible Forensic Signatures by Comprehensive TwoDimensional Gas Chromatography-Mass Spectrometry and Chemometrics, In preparation.

[30] NIST/EPA/NIH. NIST Mass Spectral Library; National Institute of Standards and Technology, U.S. Secretary of Commerce, U.S. Government Printing Office: Washington, DC, 2005. 


\section{Figures}
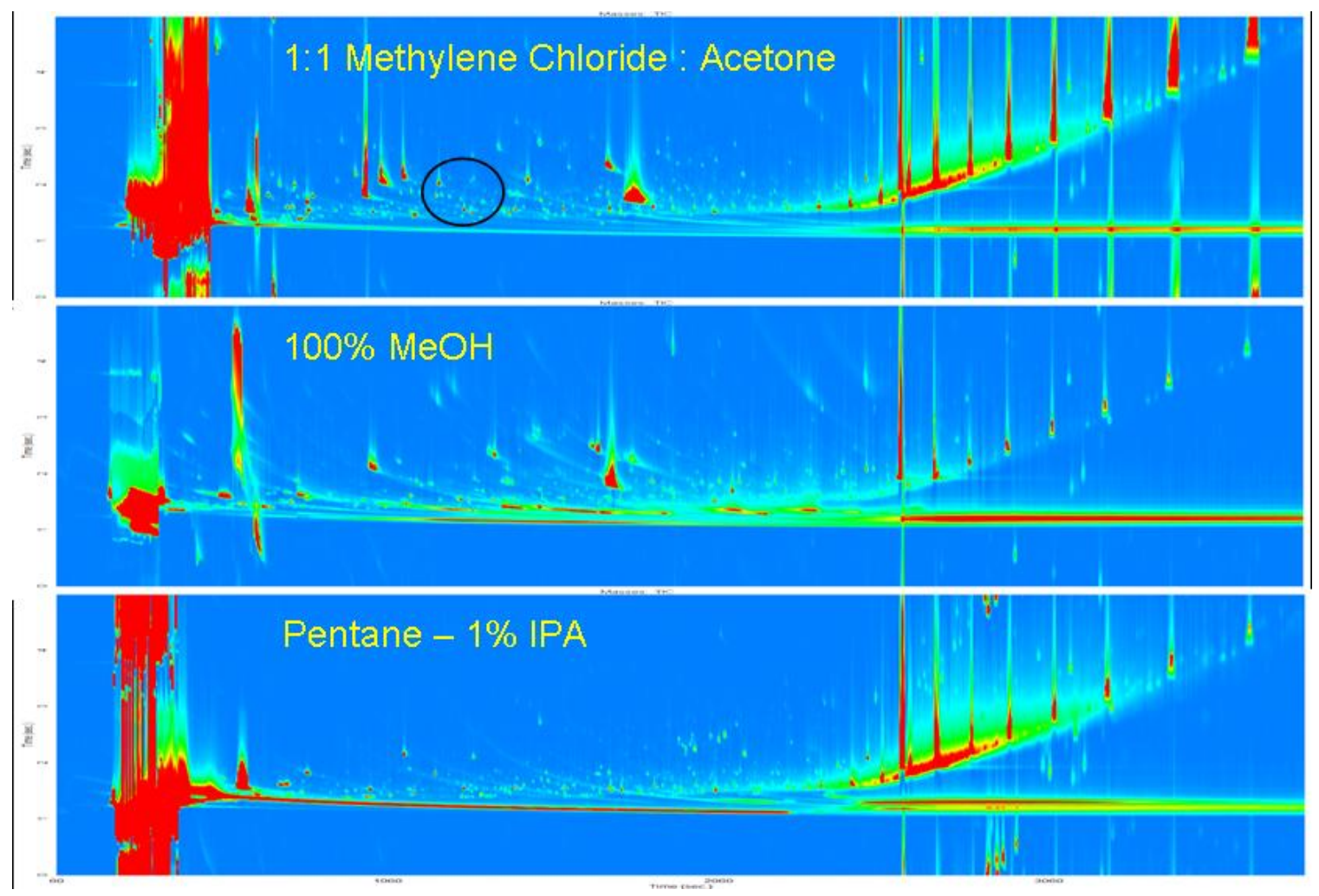

Figure 1 Representative 2D total ion chromatograms (TICs) obtained from extracted PWB for the three solvent systems listed. Within the chromatograms depicted the blue color represents the baseline signal and the red represents higher signal values. The signal intensity for the three chromatograms is set to $2 \times 10^{6}$. The large solvent zone from the splitless injection is shown early in the separations, where the methanol solvent zone appears less due to the mass spectral acquisition settings not capturing the entire mass spectrum of methanol. The circled region show in the 1:1 methylene chloride : acetone extract highlights the region where the four target compounds elute. 


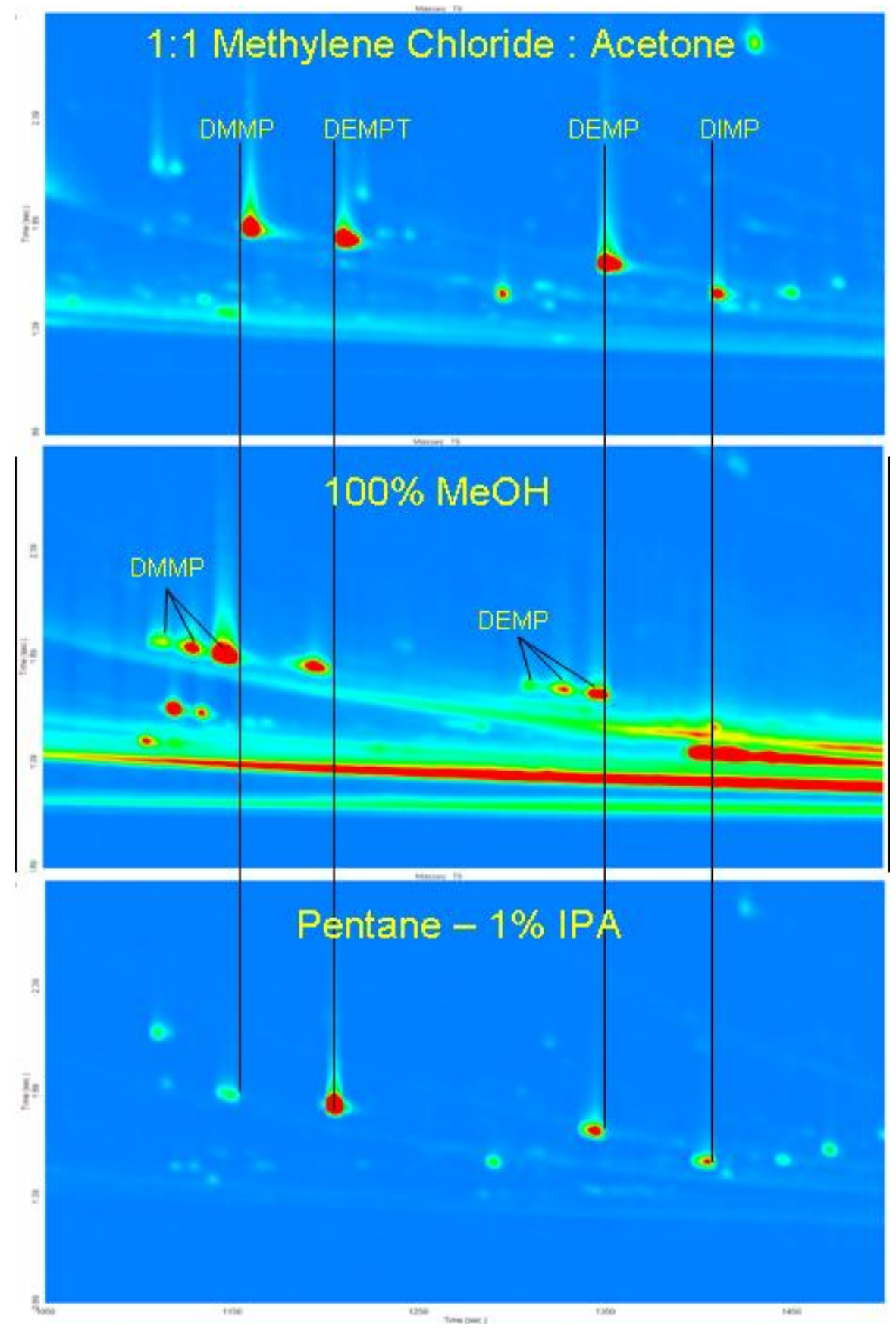

Figure 2 Representative 2D chromatograms reconstructed utilizing $m / z=79$ for clarity from the targeted region in Figure 1. The signal intensity for the three chromatograms is set to $3 \times 10^{4}$. In general the four target compounds can easily identified within the separation for all the solvent systems examined. However, when 100\% methanol is utilized DMMP and DEMP show multiple chromatographic zones (according to the mass spectral information), which is likely due to a solvent-solute-stationary phase incompatibility. 


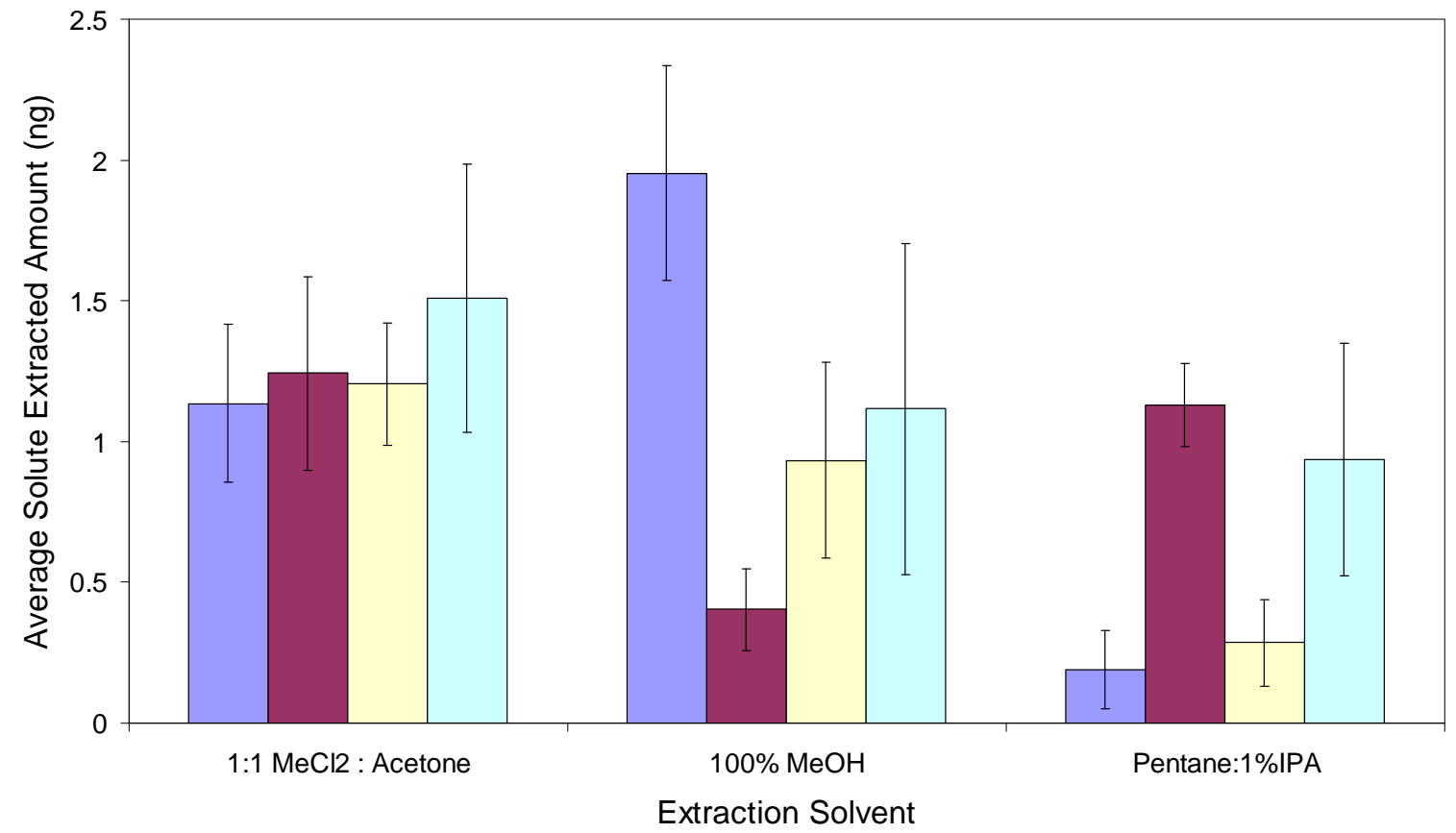

Figure 3 Summary of the extraction efficiency obtained for the four compounds illustrated as a function of the extraction solvent. The extraction results are calculated utilizing the summed mass spectral ion signal intensities $m / z$ values $=79+94: 97+124+125+168$, which cover the mass spectral ions of the target compounds. Four point calibration curves for each component were utilized to determine extracted amount. The 1:1 methylene chloride : acetone solvent system depicts the most consistent extraction among the four compounds, with an average extraction efficiency of greater than $60 \%$. 


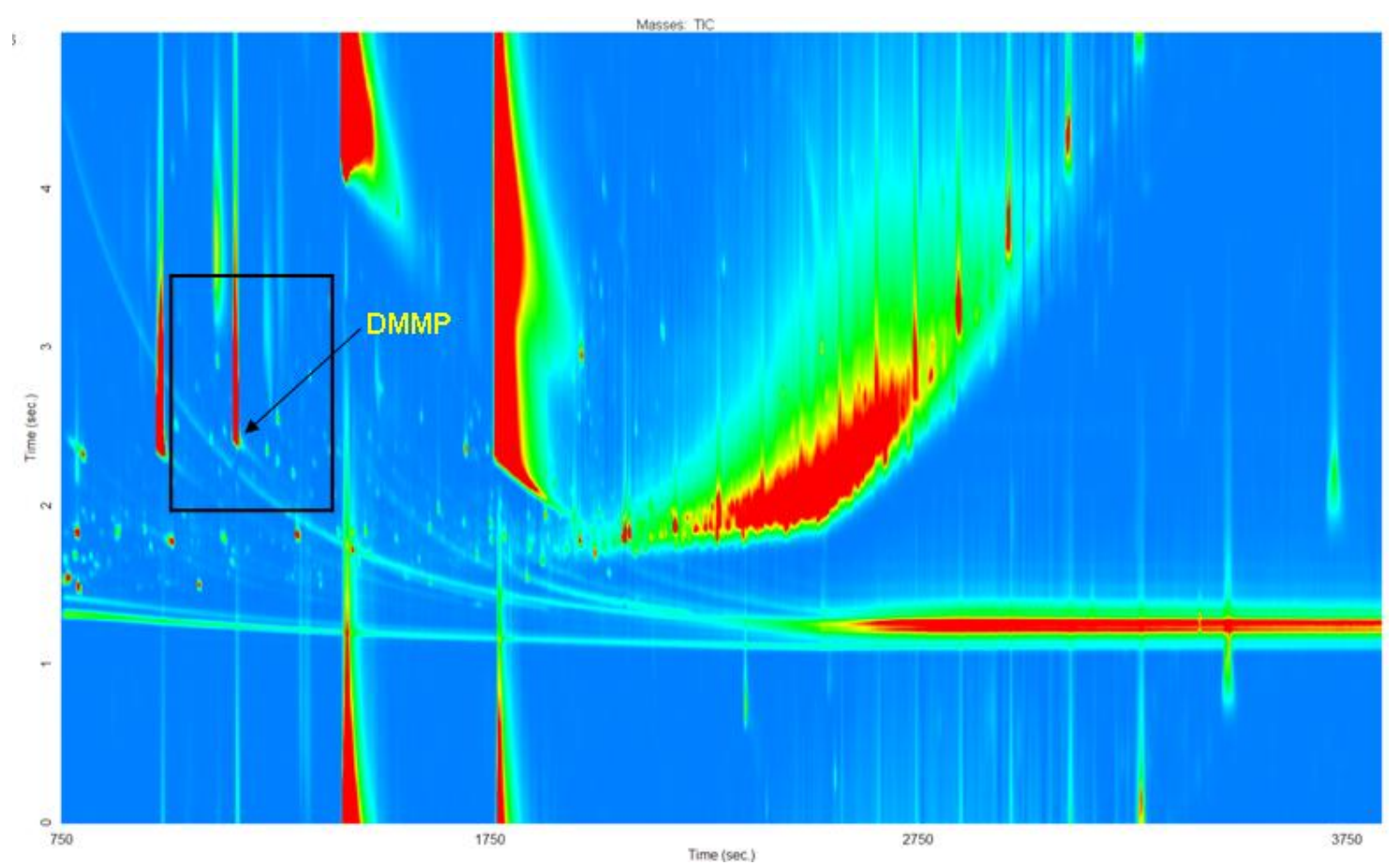

Figure 4 Representative 2D total ion chromatograms (TIC) obtained from the 1:1 methylene chloride : acetone extraction of the DMMP vapor exposed PWB sample (SNL wall board ticket). The rectangular area around DMMP solute zone highlights the region where impurity components associated with neat DMMP are found. 

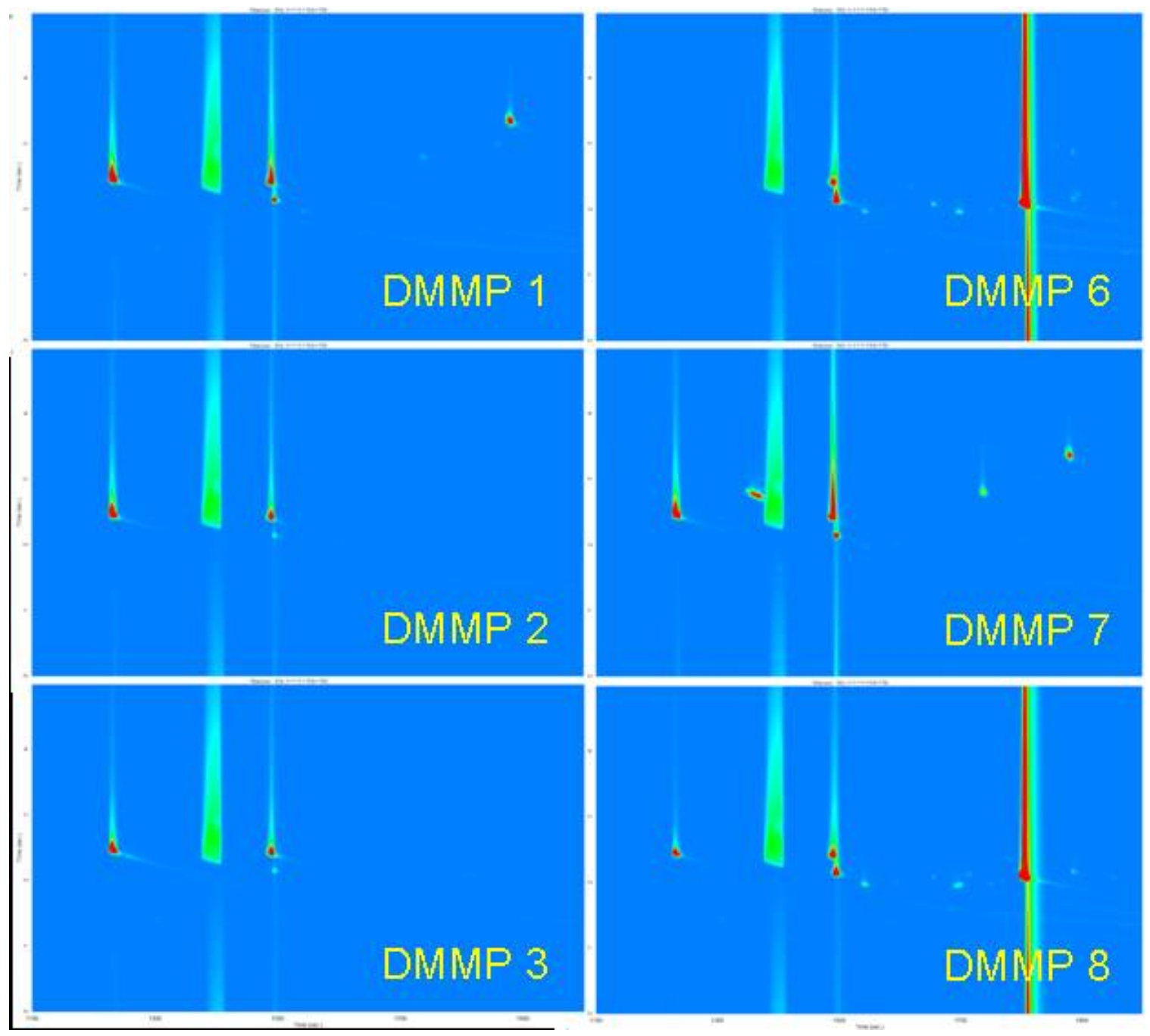

Figure 5 Highlighted chromatographic region from six commercial DMMP samples illustrated utilizing the summed $m / z$ values, $m / z=80 \times 0.05+111+154+156$, to underscore the uniqueness (based upon the presence or absence of solute zones) between samples. The signal intensity for the six chromatograms is set to $1 \times 10^{5}$. With the exception of DMMP 2 and DMMP 3 , which appear to be the same sample (op.cit. reference 29), unique impurities present in the DMMP sources can be utilized to differentiate the samples. 


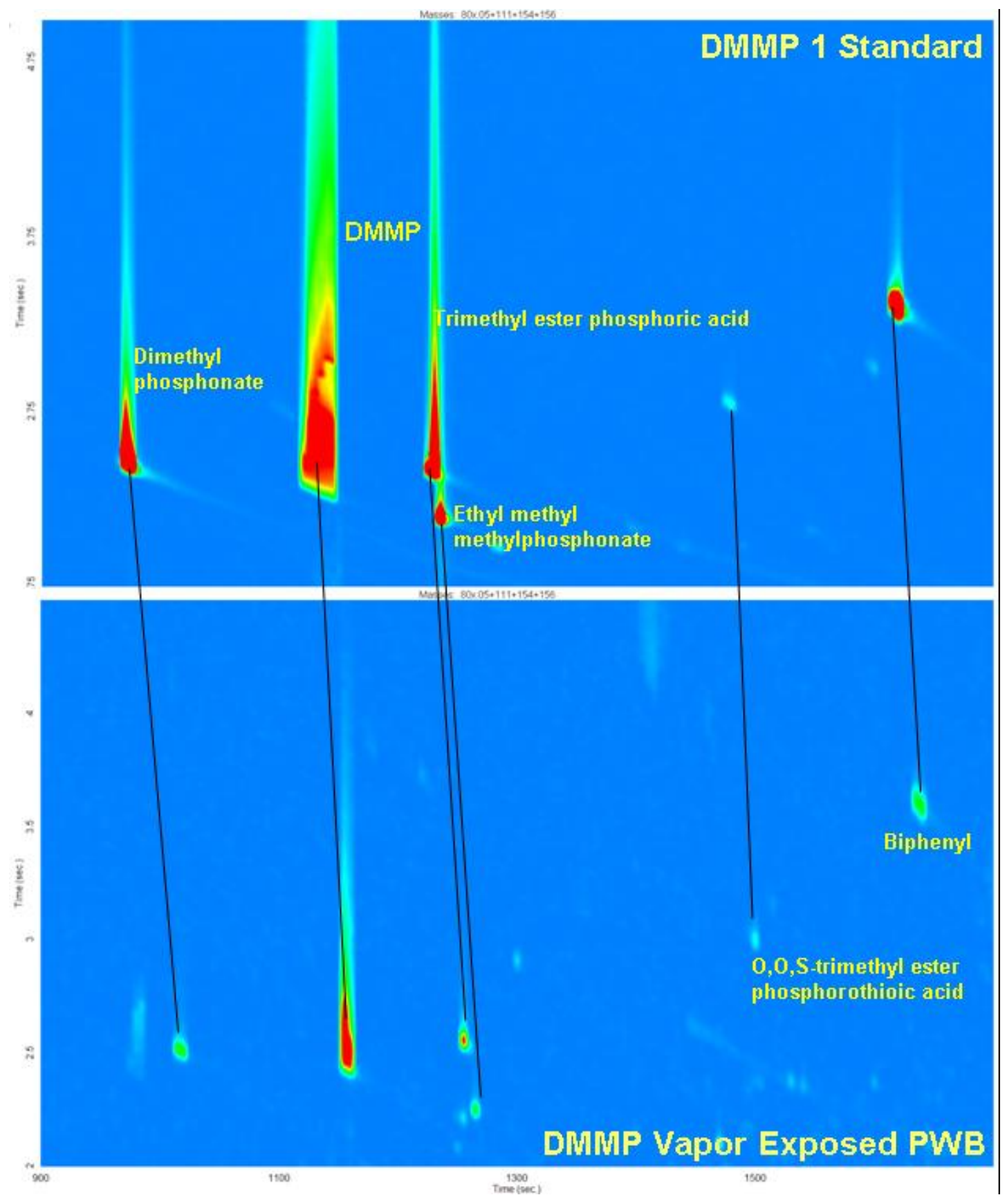

Figure 6 A chromatographic comparison between the commercial DMMP 1 sample and the extracted SNL PWB illustrated utilizing the summed $\mathrm{m} / \mathrm{z}$ values, $\mathrm{m} / \mathrm{z}=80 \mathrm{x} 0.05+111+154+156$. The signal intensity for the two chromatograms is set to $3 \times 10^{4}$. The chemical impurities, dimethyl phosphonate, trimethyl ester phosphoric acid, ethyl methyl methylphosphonate, O,O,S-trimethyl ester phosphorothioic acid, and biphenyl were found consistent between the two chromatograms based upon their 2D retention pattern and their mass spectral information. 



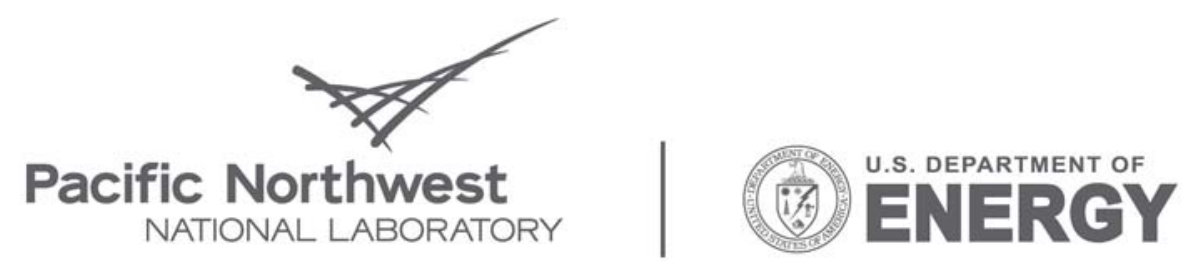

Proudly Operated by Battelle Since 1965

902 Battelle Boulevard

P.O. Box 999

Richland, WA 99352

1-888-375-PNNL (7665)

www.pnl.gov 